Raf. J. Sci.,Vol.28, No.2 Special Issue for the Third Scientific Conference of Chemistry, pp.90-99, 2019

\title{
Synthesis of Schiff Bases Derivatives from Benzene Diamine Containing Acridine Moiety
}

\author{
Shaimaa Y. Ibraheem Rafid K. Jameel \\ Department of Chemistry/ College of Science/ University of Mosul
}

(Received 13/8/2018; Accepted 25/10/2018)

\begin{abstract}
One side of ( $o, m$ and $p)$ benzene diamine reacted with aromatic aldehyde to give Schiff bases (Ia-d) or (IIa-d) and (IIIa-d) respectively. The second amine group of the synthesized Schiff bases (Ia-d or IIa-d and IIIa-d) was reacted with substituted-9-chloroacridine to give the final compounds namely N-(Substituted benzylidene )-N'-(Substituted acridine -9-yl) (1,2 or 1,3 and 1,4) benzene diamine (V,VI,VII). The reaction progress was followed by thin layer chromatography (TLC), and $\mathrm{R}_{\mathrm{f}}$ values were recorded. I.R. Spectral data for all the synthesized derivatives were reported. The U.V-Vis., and the ${ }^{1} \mathrm{HNMR}(300$ and $400 \mathrm{MHz})$ were recorded for some derivatives to support the structure of the synthesized compounds.
\end{abstract}

Keywords: Acridine, Benzenediamine, Schiff bases.

\section{تحضير طشقل قواعشيف من البنزن شنائي أمين الحاوية عل مجموعة أكربين}

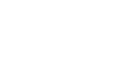

تمت مفاعلة مجموعة الأمين الأوله في الـ ـ (p, m, o) بنزين شنائي أمين مع ألديهيدات أروماتية لتطي قواعدشيف (Ia-d) أو (IIa-d) و) (IIIa-d) أما مجموعة الأمينو الثانية في قواعدشيف (Ia-d) أو (IIa-d) و(IIIa-d) فقد تمت مفاعلتها

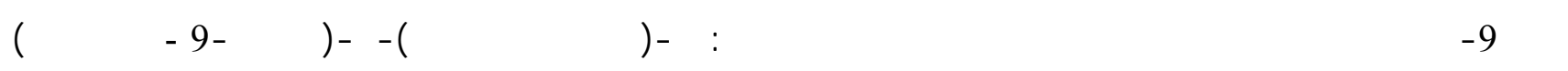

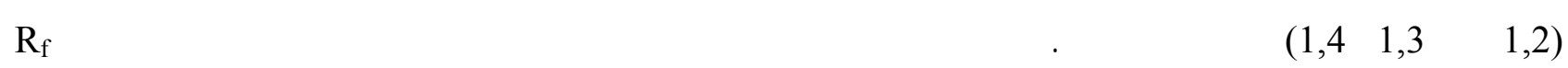

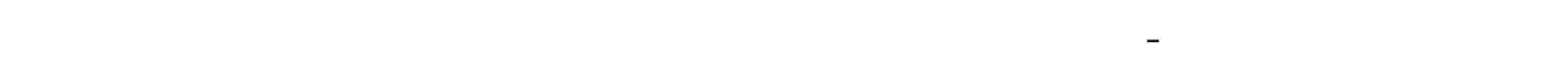
النووي المغنلطيسي (400 or 300) ميغا هرتز لدعم تشخيص هذه المركبلت.

الكامل الدالة: لٔكرينين، بذزين ثنائي أمين، قواعدشيف.

\section{INTRODUCTION}

Benzenediamine isomers are low to high toxic (Chong et al., 2016), allergy (Ed Rietchel et al., 2001) and used in hair dye (Al-Suwaidi et al., 2010).

Many chemist researcher groups focused their attention on the development of Schiff bases (Gupta et al., 2003) (El Ajaily et al., 2013) (Boghaei et al., 2002) and their metal complex (Genjal et al., 2004) (Gaballa et al., 2007), that possess cytotoxic activity (Fasina et al., 2014). Due to the former notes, these complexes used as microbial active compounds (Benguzzi, 2007), (Hamil et al., 2009), ( Fasina et al., 2012).

Condensation of 1,2-diamine compounds with carboxylic acids, dimethyl oxalate affords benzimidazole (Al Aqeel, 2017) and quinoxaline derivatives (Shekouhy et al., 2008) respectively, which are used as wide range pharmaceutical agents. 
Acridine and acridine derivative are reviewed (Kowalewska et al., 2017) and have been extensively explored as potential therapeutic agents for the treatment of a number of diseases, such as cancer (Pethy et al., 2008), Alzheimer's (Rodrigue, 2006), and as antimicrobial (Panda and Nayak, 2009), and antimalarial agents (Tomar et al., 2010). Their mode of action is mainly attributed to DNA interaction and the subsequent effects on the biological processes linked to DNA and its related enzymes (Lang et al., 2013). The above shortcomings have motivated our search and also in continuation to our work (Al-Luhaibe, 2013). Attempts have made to widening the scope and hence to synthesize some new Schiff base derivates from three of Benzene-diamine (1,2 or 1,3 and 1,4$)$ isomers containing acridine moiety. The synthesized derivatives are expected to be highly potential biologically active.

\section{EXPERIMENTAL}

All of the chemicals, solvent and reagents used of Purim grade (Note: simple or under vaccum distillation or recrystalization are some time carried out to purity chemicals). The purity of synthezied derivatives including intermediate was tested by thin layer chromatography (TLC) using silica gel (60-100 mesh), chromatography was eluted using a mixture of chloroform-methanol (9.5:0.5), or chloroform-ethyl acetate (5:5) and the spots were visualized by exposure to iodine vapour.

All melting points were uncorrected and determined by Electro thermal IA9000 Digital Melting apparatus. Complete spectra analysis was established to the synthesized derivatives. The U.V/Vis. absorbance recorded on A JASCOV - $630 \mathrm{UV} \mathrm{/} \mathrm{Vis} \mathrm{spectrophotometer} \mathrm{(Iraq,} \mathrm{Mosul}$ university, college of science). The I.R spectra were recorded on Alpha-Bruker ATR-FTIR(Iraq, Mosul, pharmacy college). ${ }^{1} \mathrm{HNMR}$ were recorded on Bruker Ultra shield $300 \mathrm{MHz}, 400 \mathrm{MHz}$ (Turkey, Gaze Entape Univ.) using DMSO-d6, as solvent with TMS as internal standard.

General procedure for synthesis of $\mathrm{N}$-(Sub. benzylidene) 1,2- benzene diamine(Ia-d) (Pati, S., 1970):

Equimolar of 1,2- benzene diamine (0.002mole) $(0.216 \mathrm{~g})$ and appropriate aromatic aldehyde (0.002 mole) dissolved and mixed in dry methanol (one drop of acetic acid was added if needed). The mixture was heated under reflux in a water bath for 3-5 hrs. and water was added to the crude product remained after solvent evaporation. The solid product was filtered off and washed several time.

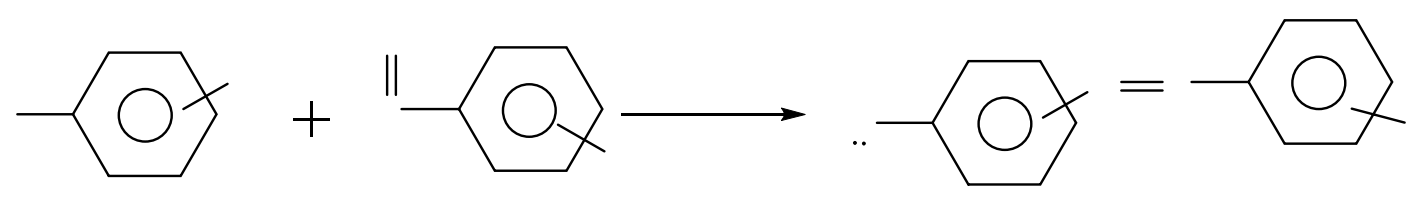

Scheme (1) 


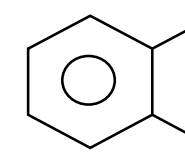<smiles>[GeH2]=[Te]</smiles><smiles>[3H]CC</smiles>

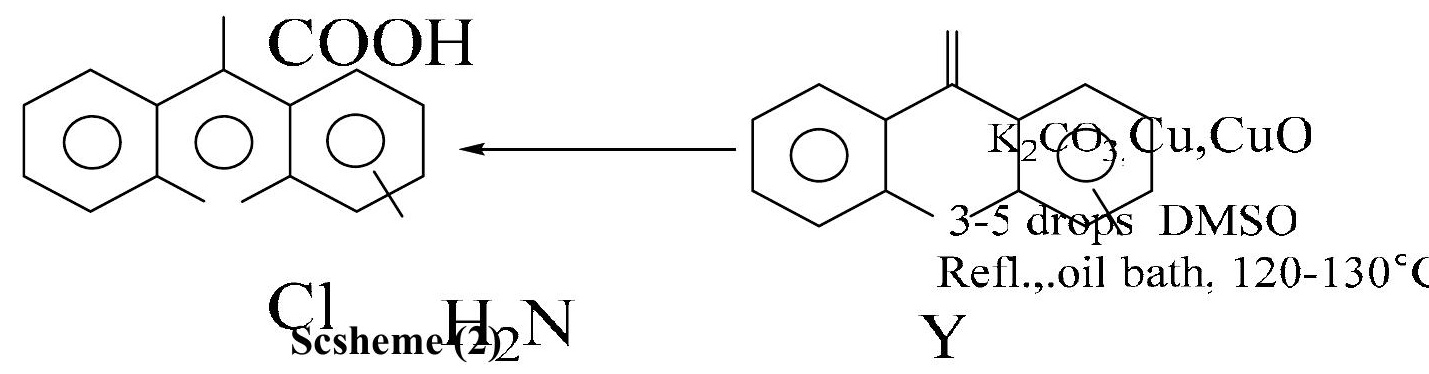<smiles>Cc1ccc2cc3ccccc3cc2c1C#Cc1ccccc1</smiles>

Scheme (3)

times with child water-methanol mixture (Note : wash with petroleum ether 40-60, if required).

The above procedure was used to prepare N-(sub.benzylidene-1,3- benzene diamine(IIa-d) and $\mathrm{N}$-(sub.benzylidene-1,4- benzene diamine(IIIa-d). Tables (1, 2 and 3) show the physical and spectral data for the compounds ( Ia-d; IIa-d; IIIa-d), respectively.

Table 1: The physical and spectral data for the Schiff bases are derived from 1,2- benzene diamine ( Ia-d)

\begin{tabular}{|c|c|c|c|c|c|c|c|}
\hline $\begin{array}{l}\text { Compd. } \\
\text { No. }\end{array}$ & $\mathbf{R}$ & $\begin{array}{c}\text { yield } \\
\%\end{array}$ & color & $\underset{{ }^{\mathbf{m}} \mathbf{C}}{{ }^{\circ} \mathbf{P}}$ & $\mathbf{R}_{\mathbf{f}}$ & $\begin{array}{c}\text { IR }\left(\mathrm{cm}^{-1}\right) \\
\mathrm{NH}_{2}, \mathrm{C}=\mathrm{N}, \text { Others }\end{array}$ & $\begin{array}{c}{ }^{1} \mathrm{HNMR}, \delta \text { ppm } \\
\text { Ar, } \mathrm{NH}_{2}, \mathbf{N}=\mathbf{C H}, \\
\mathbf{R}, \mathbf{Y}\end{array}$ \\
\hline Ia & 4-OMe & 96 & $\begin{array}{c}\text { Pale } \\
\text { orange }\end{array}$ & 74-76 & 0.50 & $\begin{array}{c}3340,1590 \\
\left(\mathrm{OCH}_{3}\right)=1242,1164\end{array}$ & \\
\hline $\mathrm{Ib}$ & $2-\mathrm{Cl}$ & 76 & Brown & $\begin{array}{l}137- \\
140\end{array}$ & 0.60 & $3528,1624,(\mathrm{C}-\mathrm{Cl}) 736$ & \\
\hline Ic & $\begin{array}{c}=4- \\
\mathrm{N}(\mathrm{Me})_{2}\end{array}$ & 98 & $\mathrm{Br}$ & & Q. & $\left.3963 y^{4}-f\right)$ & Dry-Methano \\
\hline Id & $4-\mathrm{Cl}$ & & & & & $1624,(\mathrm{C}-\mathrm{Cl}) 753$ & $\begin{array}{c}\text { 7.0ed.5. } 5 \text { (dit), hrss } \\
6.85\end{array}$ \\
\hline
\end{tabular}


Table 2: The physical and spectral data for the Schiff bases are derived from 1,3- benzene diamine( IIa-d)

\begin{tabular}{|c|c|c|c|c|c|c|c|}
\hline $\begin{array}{l}\text { Compd. } \\
\text { No. }\end{array}$ & $\mathbf{R}$ & $\begin{array}{l}\text { yield } \\
\%\end{array}$ & color & $\underset{{ }^{\circ} \mathbf{C}}{\mathbf{m} . \mathbf{p}}$ & $\mathbf{R}_{\mathbf{f}}$ & $\begin{array}{c}\operatorname{IR}\left(\mathrm{cm}^{-1}\right) \\
\mathrm{NH}_{2}, \mathrm{C}=\mathrm{N}, \text { Others }\end{array}$ & $\begin{array}{c}{ }^{1} \mathrm{HNMR}, \delta \mathrm{ppm} \\
\mathrm{Ar}, \mathrm{NH}_{2}, \mathrm{~N}=\mathrm{CH}, \mathrm{R}, \mathrm{Y}\end{array}$ \\
\hline IIa & 4-OMe & 40 & $\begin{array}{c}\text { Dark } \\
\text { red }\end{array}$ & $78-80$ & 0.60 & $3340,1590,\left(\mathrm{OCH}_{3}\right) 1242$ & $\begin{array}{c}7.02-9.85(8 \mathrm{H}), 5.08 \\
6.89,3.3\left(\mathrm{OCH}_{3}\right) \\
\end{array}$ \\
\hline $\mathrm{IIb}$ & $\begin{array}{l}2,3-\mathrm{di} \\
(\mathrm{OMe})\end{array}$ & 74 & Brown & $\begin{array}{l}165- \\
167\end{array}$ & 0.49 & $\begin{array}{c}3340,1616, \\
\left(\mathrm{OCH}_{3}\right) 1263,1227\end{array}$ & - \\
\hline IIc & $2-\mathrm{Cl}$ & 94 & $\begin{array}{c}\text { Dark } \\
\text { Brown }\end{array}$ & $\begin{array}{l}170- \\
172\end{array}$ & 0.31 & $\begin{array}{c}3410,1604, \\
(\mathrm{C}-\mathrm{Cl}) 730\end{array}$ & \\
\hline IId & $4-\mathrm{N}(\mathrm{Me})_{2}$ & 88 & Black & $83-85$ & 0.67 & 3188,1581 & $\begin{array}{c}6.35-9.65(8 \mathrm{H}), 3.25 \\
6.39,2.85\left(2 \mathrm{CH}_{3}\right), 2.90 \\
\end{array}$ \\
\hline
\end{tabular}

Table 3: The physical and spectral data for the Schiff bases are derived from 1,4- benzene diamine( IIIa-d)

\begin{tabular}{|c|c|c|c|c|c|c|c|}
\hline $\begin{array}{l}\text { Compd. } \\
\text { No. }\end{array}$ & $\mathbf{R}$ & $\begin{array}{c}\text { yield } \\
\%\end{array}$ & color & $\underset{{ }^{\circ} \mathbf{C} . \mathbf{P}}{\mathbf{o}^{\circ}}$ & $\mathbf{R}_{\mathbf{f}}$ & $\begin{array}{c}\mathrm{IR}\left(\mathrm{cm}^{-1}\right), \mathrm{NH}_{2}, \\
\mathrm{C}=\mathbf{N}, \text { Others }\end{array}$ & $\begin{array}{r}{ }^{1} \mathrm{HNMR} \delta \mathrm{ppm} \\
\text { Ar.,NH }, \mathrm{N}=\mathrm{CH}, \mathbf{R}, \mathrm{Y}\end{array}$ \\
\hline IIIa & 4-OMe & 97 & Yellow & $\begin{array}{c}139- \\
141\end{array}$ & $0.89^{*}$ & $\begin{array}{l}3040,1592, \\
\left(\mathrm{OCH}_{3}\right) 1244\end{array}$ & ------------- \\
\hline IIIb & $\begin{array}{l}2,3-\mathrm{di} \\
(\mathrm{OMe})\end{array}$ & 96 & Orange & $\begin{array}{l}127- \\
128\end{array}$ & 0.40 & $\begin{array}{c}3373,1620 \\
\left(\mathrm{OCH}_{3}\right) 1267 \\
1217\end{array}$ & $\begin{array}{c}7.54-8.91(7 \mathrm{H}), \\
3.85,7.39, \\
2.48,3.34\left(2 \mathrm{OCH}_{3}\right)\end{array}$ \\
\hline IIIc & $4-\mathrm{N}(\mathrm{Me})_{2}$ & 88 & Yellow & $\begin{array}{l}259- \\
260\end{array}$ & 0.40 & 3438,1586 & -------------- \\
\hline IIId & 2- $\mathrm{NH}_{2}$ & 90 & black & $97-99$ & 0.80 & $\begin{array}{l}3122,1606, \\
\left(\mathrm{NH}_{2}\right) 3235\end{array}$ & $\begin{array}{c}5.95-7.60(8 \mathrm{H}), 5.01, \\
5.69,5.43\left(\mathrm{NH}_{2}\right)\end{array}$ \\
\hline
\end{tabular}

$*$ Et Ac: $\mathrm{CHCl}_{3}(5: 5)$

General procedure for synthesis 9-chloro subs. acridine (IVa-f) (Acheson and Bottom, 1975), (Mahmood, 2013)

To $50 \mathrm{ml}$ of thionyl chloride $\left(\mathrm{SOCl}_{2}\right)$ (2-3 drop DMF) was added in round bottom flask, equiped by double surface condenser supported with $\mathrm{CaCl}_{2}$ tube, $3 \mathrm{~g}$ of subs. Acridone was added gradually with in (1-2 hrs) with continuous stirring. The stirring and reflux were continued in water bath $\left(60-70^{\circ}\right)$ for $3 \mathrm{hrs}$. The reaction mixture finally converted to clear orange or sometimes dark brown. The access of thionyl chloride was distilled off under vacuum. The crude product was added slowly to conc. Ammonia solution: ice mixture and digested for $1 \mathrm{hr}$. The basic mixture was extracted 3 times with $25 \mathrm{ml}$ of chloroform. The collected organic layer was dried with magnisum sulfate (for over night then), Filtered off and distilled under vacuum. The solid product is pure enough to continue the next step. (Note: ammonia: ethanol mixture was used as a solvent of crystallization if needed) (Mahmood 2013). The physical constants were listed in (Table 4).

Table 4: The physical constants of 9-chloro acridine

\begin{tabular}{|c|c|c|c|c|c|}
\hline $\begin{array}{c}\text { Compd. } \\
\text { No. }\end{array}$ & $\mathbf{R}$ & $\begin{array}{c}\text { Yield } \\
\mathbf{\%}\end{array}$ & Color & $\begin{array}{c}\mathbf{m . p} \\
{ }^{\circ} \mathrm{C}\end{array}$ & $\mathbf{R}_{\mathbf{f}}$ \\
\hline $\mathrm{IVa}$ & $2-\mathrm{Br}$ & 46 & Orange & Oily & 0.15 \\
\hline $\mathrm{IV}$ & $2-\mathrm{Me}$ & 76 & Orange & $92-94$ & 0.32 \\
\hline $\mathrm{IVc}$ & $4-\mathrm{Me}$ & 89 & Orange & $76-78$ & 0.76 \\
\hline $\mathrm{IVd}$ & $2-\mathrm{OMe}$ & 58 & Dark orange & $84-87$ & 0.32 \\
\hline $\mathrm{IVe}$ & $4-\mathrm{OMe}$ & 74 & Orange & $70-73$ & 0.20 \\
\hline $\mathrm{IVf}$ & $4-\mathrm{NO}_{2}$ & 71 & Dark orange & Oily & 0.25 \\
\hline
\end{tabular}


General procedure for synthesis of $\mathrm{N}$-(subs. benzylidene)-N'-(subs.acridin-9-yl) 1,2- benzene diamine

Equimolar (0.001mole) of subs.-9-chloroacridine (IVa-f) was mixed with Schiff base (Ia-d or IIa-d and IIIa-d) in $(30 \mathrm{ml})$ of dry methanol. The reaction mixture was heated under reflux at least for $10 \mathrm{hrs}$. TLC monitor was at the end of the reaction. The solvent was removed by simple distillation, and crude product was washed with $(10 \mathrm{ml})$ sodium bicarbonate $(10 \%)$. The crude solid was washed with water then air dried .The product was washed several times with petroleum Ether (40-60) then dried under vacuum. This procedure was used to synthesize the 1,3 and 1,4 benzene diamine derivatives. Tables $(5,6$ and 7$)$ show the physical constants of these derivatives.

Table 5: The physical constants of $\mathrm{N}$-(substituted benzylidene)- $\mathrm{N}^{-}$-(substituted acridin-9-yl) 1,2 benzene diamine.

\begin{tabular}{|c|c|c|c|c|c|c|}
\hline $\begin{array}{c}\text { Compd. } \\
\text { No. }\end{array}$ & $\mathbf{Y}$ & $\mathbf{R}$ & $\begin{array}{c}\text { Yield } \\
\mathbf{\%}\end{array}$ & Color & $\begin{array}{c}\mathbf{m} . \mathbf{p} \\
{ }^{\circ} \mathrm{C}\end{array}$ & $\mathbf{R}_{\mathbf{f}}$ \\
\hline $\mathrm{Va}$ & $2-\mathrm{Br}$ & $4-\mathrm{N}\left(\mathrm{CH}_{3}\right)_{2}$ & 86 & Reddish Orange & $79-81$ & 0.24 \\
\hline $\mathrm{Vb}$ & $2-\mathrm{Br}$ & $2-\mathrm{Cl}$ & 83 & Black Orange & $80-82$ & 0.53 \\
\hline $\mathrm{Vc}$ & $4-\mathrm{CH}_{3}$ & $4-\mathrm{Cl}$ & 83 & Orange & $62-64$ & 0.73 \\
\hline $\mathrm{Vd}$ & $4-\mathrm{CH}_{3}$ & $\left.4-\mathrm{N}_{(\mathrm{CH}}\right)_{2}$ & 89 & Orange & $149-151$ & 0.59 \\
\hline $\mathrm{Ve}$ & $2-\mathrm{CH}_{3}$ & $4-\mathrm{OCH}_{3}$ & 88 & Red & $57-58$ & 0.44 \\
\hline $\mathrm{Vf}$ & $2-\mathrm{CH}_{3}$ & $4-\mathrm{N}_{3}\left(\mathrm{CH}_{3}\right)_{2}$ & 86 & Brown & $103-105$ & 0.37 \\
\hline $\mathrm{Vg}$ & $2-\mathrm{OCH}_{3}$ & $4-\mathrm{OCH}_{3}$ & 89 & Orange & $54-57$ & $0.68^{*}$ \\
\hline $\mathrm{Vh}$ & $4-\mathrm{OCH}_{3}$ & $4-\mathrm{OCH}_{3}$ & 83 & Black Orange & $43-45$ & 0.77 \\
\hline $\mathrm{Vi}$ & $4-\mathrm{NO}_{2}$ & $2-\mathrm{Cl}_{3}$ & 84 & Black Orange & $71-73$ & 0.35 \\
\hline $\mathrm{Vj}$ & $4-\mathrm{NO}_{2}$ & $4-\mathrm{N}\left(\mathrm{CH}_{3}\right)_{2}$ & 87 & Orange & $138-141$ & 0.34 \\
\hline
\end{tabular}

* EtOAc: $\mathrm{CHCl}_{3}(50: 50)$

Table 6: The physical constants of $\mathrm{N}$-(substituted benzylidene)-N־-(substituted acridin-9yl) 1,3 benzene diamine

\begin{tabular}{|c|c|c|c|c|c|c|}
\hline $\begin{array}{c}\text { Compd. } \\
\text { No. }\end{array}$ & $\mathbf{Y}$ & $\mathbf{R}$ & Yield \% & Color & $\begin{array}{c}\mathbf{m} . \mathbf{p} \\
\mathbf{0}\end{array}$ & $\mathbf{R}_{\mathbf{f}}$ \\
\hline Via & $4-\mathrm{CH}_{3}$ & 2,3 -dimethoxy & 69 & Orange & $142-145$ & 0.77 \\
\hline $\mathrm{VIb}$ & $4-\mathrm{CH}_{3}$ & $2-\mathrm{Cl}$ & 63 & Dark orange & $85-87$ & 0.66 \\
\hline VIc & $2-\mathrm{CH}_{3}$ & 2,3 -dimethoxy & 97 & Pale brown & $131-133$ & $0.36^{*}$ \\
\hline VId & $2-\mathrm{CH}_{3}$ & $2-\mathrm{Cl}$ & 98 & Greenish black & $209-212$ & $0.34 *$ \\
\hline VIe & $2-\mathrm{OCH}_{3}$ & $4-\mathrm{OCH}_{3}$ & 65 & Black & $132-134$ & 0.40 \\
\hline VIf & $2-\mathrm{OCH}_{3}$ & $2-\mathrm{Cl}$ & 98 & Black & $71-73$ & 0.28 \\
\hline VIg & $4-\mathrm{OCH}_{3}$ & $2,3-$ dimethoxy & 99 & Reddish orange & $201-203$ & $0.72 *$ \\
\hline VIh & $4-\mathrm{OCH}_{3}$ & $2-\mathrm{Cl}$ & 97 & Brown & $208-210$ & 0.51 \\
\hline VIi & $4-\mathrm{NO}_{2}$ & $2,3-$-dimethoxy & 37 & Orange & Low temp. & 0.42 \\
\hline VIj & $4-\mathrm{NO}_{2}$ & 2-Cl & 48 & Brown & Low temp. & 0.64 \\
\hline
\end{tabular}

* EtOAc: $\mathrm{CHCl}_{3}(5: 5)$ 
Table 7: The physical constants of $\mathrm{N}$-(substituted benzylidene)- $\mathrm{N}^{-}$-(substituted acridin-9-yl) 1,4 benzene diamine

\begin{tabular}{|c|c|c|c|c|c|c|}
\hline $\begin{array}{c}\text { Compd. } \\
\text { No. }\end{array}$ & $\mathbf{Y}$ & $\mathbf{R}$ & $\begin{array}{c}\text { Yield } \\
\mathbf{c}\end{array}$ & Color & $\begin{array}{c}\mathbf{m . p} \\
{ }^{\circ} \mathrm{C}\end{array}$ & $\mathbf{R}_{\mathbf{f}}$ \\
\hline VIIa & $2-\mathrm{Br}$ & 4- $\mathrm{OCH}_{3}$ & 81 & Brown & $125-127$ & 0.34 \\
\hline VIIb & $2-\mathrm{Br}$ & 2,3 -dimethoxy & 83 & Gray & $117-119$ & 0.30 \\
\hline VIIc & $4-\mathrm{CH}_{3}$ & 2,3-dimethoxy & 95 & Brown & $153-155$ & 0.89 \\
\hline VIId & $2-\mathrm{CH}_{3}$ & $4-\mathrm{N}_{3}\left(\mathrm{CH}_{3}\right)_{2}$ & 96 & Pale orange & $138-141$ & 0.58 \\
\hline VIIe & $2-\mathrm{CH}_{3}$ & $4-\mathrm{OCH}_{3}$ & 96 & Brown & $148 \mathrm{~d}$ & 0.34 \\
\hline VII f & $2-\mathrm{OCH}_{3}$ & $4-\mathrm{OCH}_{3}$ & 93 & Orange & $97-100$ & $0.94 *$ \\
\hline VIIg & $4-\mathrm{OCH}_{3}$ & $4-\mathrm{OCH}_{3}$ & 98 & Orange & $180-182$ & $0.79^{*}$ \\
\hline VIIh & $4-\mathrm{OCH}_{3}$ & $4-\mathrm{N}\left(\mathrm{CH}_{3}\right)_{2}$ & 76 & Black Orange & $102-103$ & 0.71 \\
\hline VIIi & $4-\mathrm{NO}_{2}$ & $2,3-$ dimethoxy & 71 & Orange & Oily & 0.32 \\
\hline VII j & $4-\mathrm{NO}_{2}$ & 2-Cl & 86 & Orange & $84-87$ & 0.30 \\
\hline
\end{tabular}

$*$ EtOAc: $\mathrm{CHCl}_{3}(5: 5)$

Table 8: The IR and ${ }^{1} \mathrm{HNMR}$ of spectral data of some N-(substituted benzylidene)-N"(substituted acridin-9-yl)benzene diamine

\begin{tabular}{|c|c|c|}
\hline $\begin{array}{l}\text { Compd. } \\
\text { No. }\end{array}$ & $\begin{aligned} \operatorname{IR}\left(\mathrm{cm}^{-1}\right) \\
\mathrm{NH},=\mathrm{CH}, \mathrm{C}=\mathrm{N}, \text { Other }\end{aligned}$ & $\begin{array}{c}{ }^{1} \mathrm{HNMR}, \boldsymbol{\delta} \text { ppm } \\
\text { NH, Ar., N=CH,R,Y }\end{array}$ \\
\hline $\mathrm{Va}$ & $3225,2921,1596,1511$ (aromatic), $1367(\mathrm{C}-\mathrm{N}), 1454\left(\mathrm{CH}_{3}\right)$ & $\begin{array}{c}12.18,6.63-8.48(15 \mathrm{H}), 5.54,2.81- \\
3.04\left(2 \mathrm{CH}_{3}\right) \\
\end{array}$ \\
\hline $\mathrm{Vc}$ & $3047,2920,1581,1517$ (aromatic),736 (C-Cl) & $\begin{array}{c}10.66,7.27-8.80(15 \mathrm{H}), 7.17,2.61-2.69 \\
\left(\mathrm{CH}_{3}\right)\end{array}$ \\
\hline $\mathrm{Vj}$ & $3122,2920,1603,1498$ (aromatic) & $11.49,6.59-9.67(16 \mathrm{H}), 3.01\left(2 \mathrm{CH}_{3}\right)$ \\
\hline VI c & $\begin{array}{c}3321,2918,1626,1580(\operatorname{aromatic}), 1261(\mathrm{ROAr}), 1369 \text { (C- } \\
\mathrm{N})\end{array}$ & $\begin{array}{c}11.76,7.02-8.2(14 \mathrm{H}), 6.81,3.9(\mathrm{~m}) \\
\left(2 \mathrm{CH}_{3}\right)\end{array}$ \\
\hline VI e & $3340,2918,1670,1590$ (aromatic), 1242 (ROAr) & $\begin{array}{c}11.70,7.0-9.87(15 \mathrm{H}), 6.68,3.75- \\
3.96\left(2 \mathrm{OCH}_{3}\right)\end{array}$ \\
\hline VIg & $\begin{array}{c}\text { 3181, 2850, 1616,1571(aromatic), 1248(ROAr), } 1466\left(\mathrm{CH}_{3}\right), \\
1330(\mathrm{C}-\mathrm{N})\end{array}$ & $\begin{array}{l}11.23,7.08-8.31(14 \mathrm{H}), 6.88 \\
4.05,4.06,4.10\left(3 \mathrm{OCH}_{3}\right)\end{array}$ \\
\hline VIIa & $\ldots \ldots$ & $\begin{array}{c}9.87,6.68-8.63(15 \mathrm{H}), 6.59,3.65 \\
\left(\mathrm{OCH}_{3}\right)\end{array}$ \\
\hline VIId & ------------------------------' & $9.67,6.70-8.50(15 \mathrm{H}), 3.02,2.33\left(2 \mathrm{CH}_{3}\right)$ \\
\hline VII $\mathrm{f}$ & 3367, 2920, 1587 (aromatic), 1242(ROAr), 1308 (C-N) & $\begin{array}{c}9.87,6.77-8.65(15 \mathrm{H}), 6.74,3.85-3.87 \\
\left(2 \mathrm{CH}_{3}\right)\end{array}$ \\
\hline
\end{tabular}

\section{Electronic Spectra}

\section{RESULT AND DISCUSSION}

U.V and Visible spectra of finally synthesized derivatives show maximum wave length bands (Log molar absorptivity) i.e. $\lambda_{\max }(\log \varepsilon)$. The 1,2-diamine derivatives (Va-j) show $\lambda_{\max }$ of 330-340 $\mathrm{nm}, \log \varepsilon$ of (4.204-3.69), while the 1,3-diamine derivatives (VIa-j) show more shift to higher wave length but with lower absorbance i.e. $375-400 \mathrm{~nm}$ (3.78-3.40), only the derivative (VIIa) shows good visible absorbance $(430 \mathrm{~nm}, \log \varepsilon=3.95)$. The derivatives of 1,4 -diamine show two $\lambda_{\max }$ of $450 \mathrm{~nm}$ and $475 \mathrm{~nm}$ with $\log \varepsilon$ of 3.87 and 3.30 respectively while only derivative (VIId) shows $\lambda_{\max } 370, \log \varepsilon$ of 4.38 .

\section{I.R spectra}

All the synthesized compounds from (1,2-diamino) (Table 1) show that the range of $v \mathrm{NH}$, $3225-3065 \mathrm{~cm}^{-1}, v=\mathrm{CH}$ at $2918-2922 \mathrm{~cm}^{-1}, v \mathrm{C}=\mathrm{N}$ at $1632-1584 \mathrm{~cm}^{-1}$; these data are quite similar to that of I.R standard charesterectic groups. For 1,3-diamine derivatives (Table 2) show $v \mathrm{NH}$ at the range $3340-3045 \mathrm{~cm}^{-1}, v=\mathrm{CH}$ at range $2919-2848 \mathrm{~cm}^{-1}$, also $\vee \mathrm{C}=\mathrm{N}$ at the range $1626-1616 \mathrm{~cm}^{-1}$, which are in very good manner of characteristic group of I.R. For 1,4-diamine derivatives. 
(Table 3) shows $v$ NH at 3386-3199 $\mathrm{cm}^{-1}, v=\mathrm{CH} 2919-2830 \mathrm{~cm}^{-1}$, also to $v \mathrm{~N}=\mathrm{C}$ at $1632-1585$ $\mathrm{cm}^{-1}$. these data are good support to the foundation of characteristic groups (Parikh, V.M. 1974).

${ }^{1}$ HNMR spectra

${ }^{1} \mathrm{HNMR}$ data excellently prove the synthesized compounds, (Table 8 ). shows the $\delta$ ppm of $(\mathrm{NH}),(\mathrm{H})$ aromatic, $=\mathrm{CH}$, and other substituents $\mathrm{CH}_{3}, \mathrm{OCH}_{3}$, ...etc. these data were in agreement with literature and previous work (Sondhi et al., 1997), (Sondhi et al., 2004), (Mahmood, 2013), Fig (1-6).

\section{ACKNOWLEDGEMENTS}

The authors are thankful to the College of Sciences of the University of Mosul for study vacation for Shaimaa Y. Ibraheem. Also thanks go to Dr. S. K. Younis and Mr. M.AL-rawi for NMR measurement.

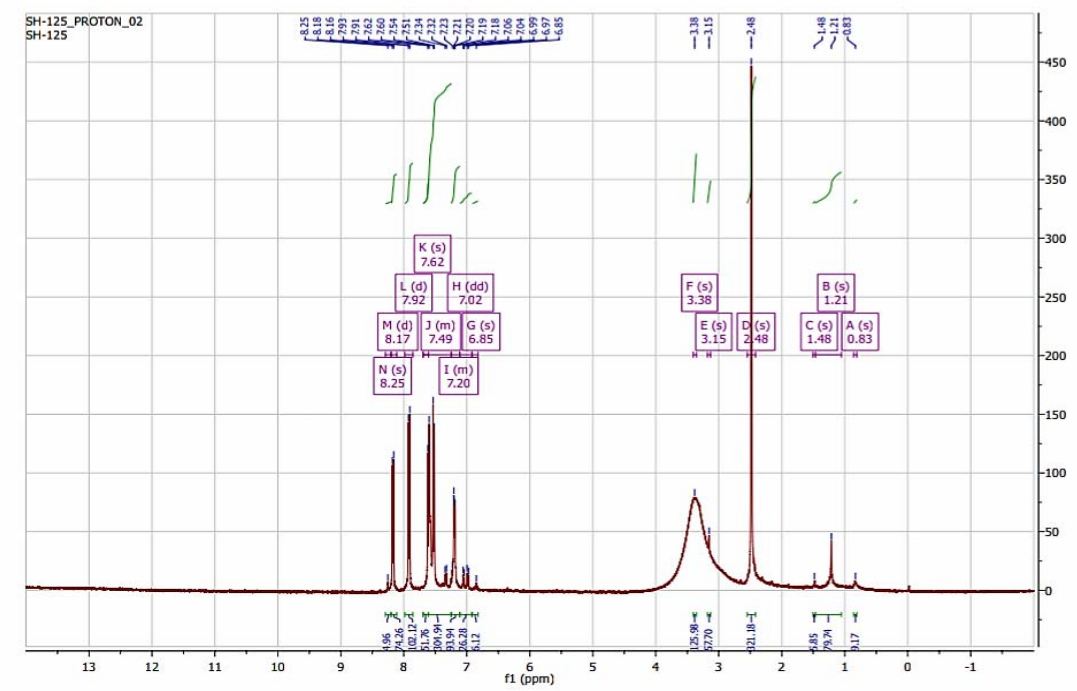

Fig. 1: ${ }^{1}$ HNMR spectrum for compound (Id)

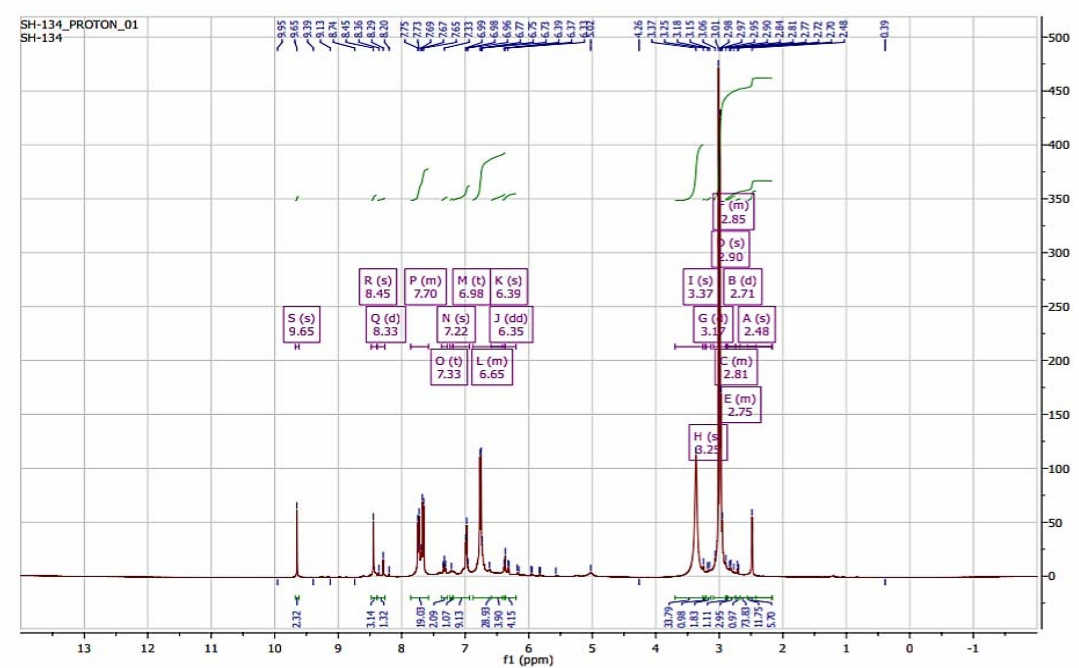

Fig. 2: ${ }^{1}$ HNMR spectrum for compound (IId) 


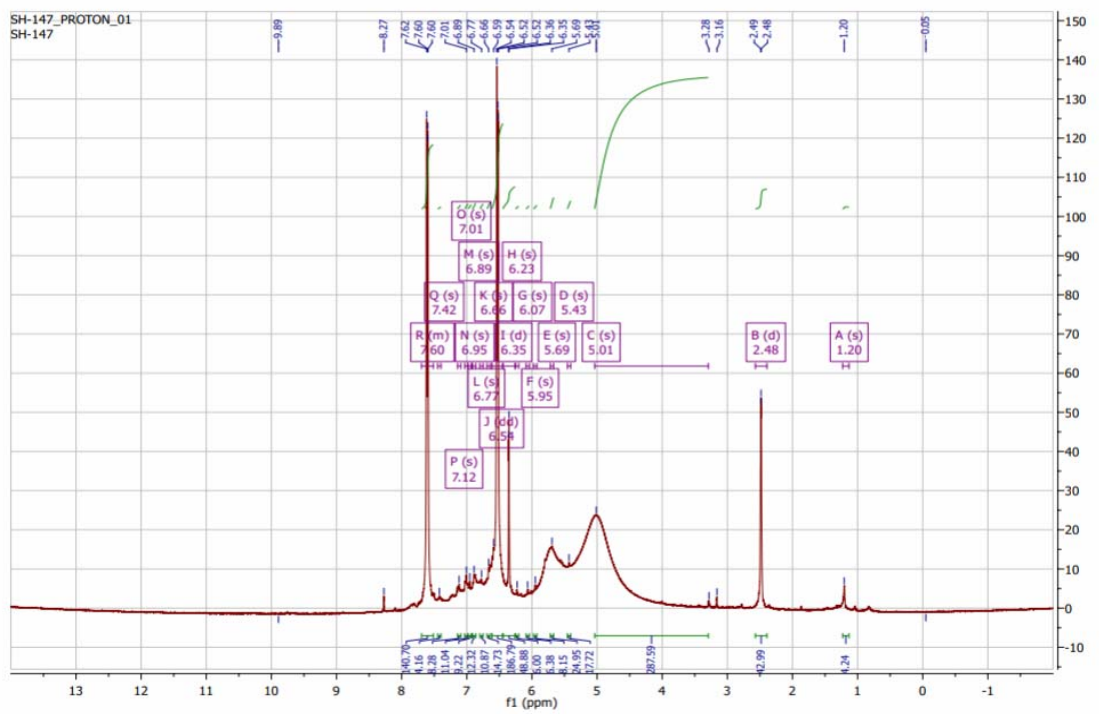

Fig. 3: ${ }^{1}$ HNMR spectrum for compound (IIId)

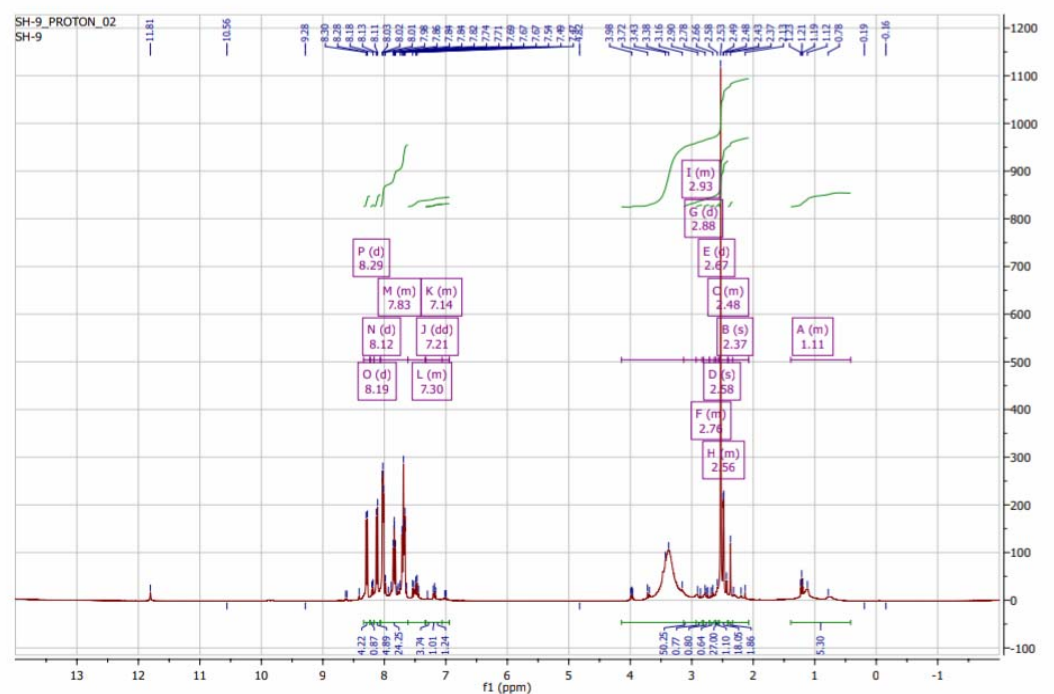

Fig. 4: ${ }^{1}$ HNMR spectrum for compound (IVb)

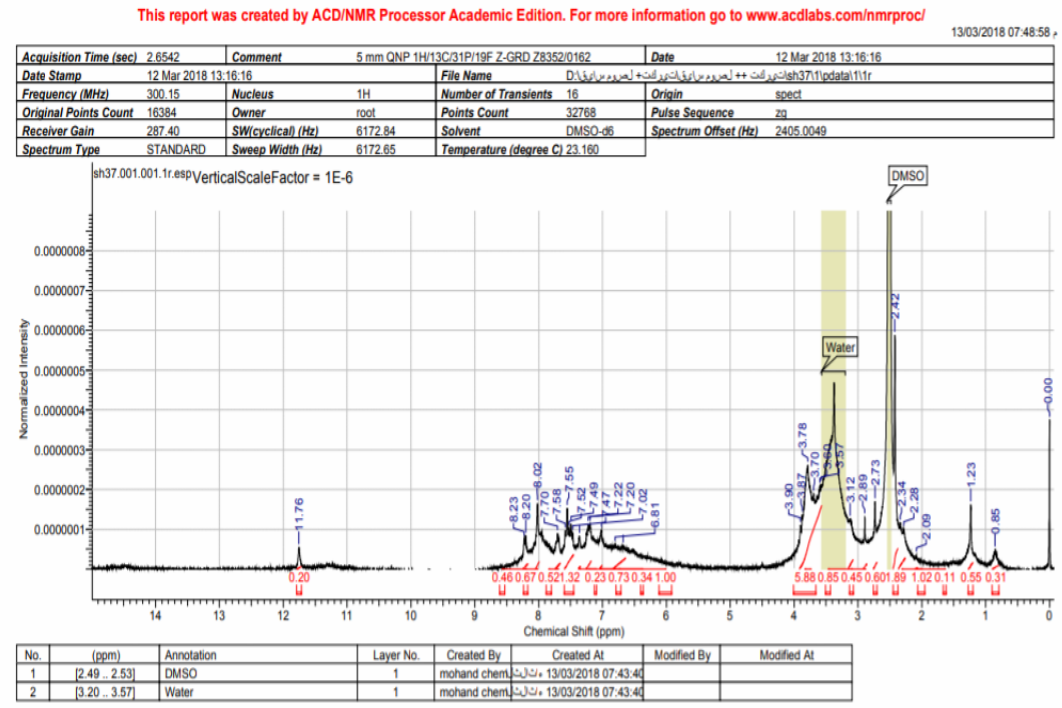

Fig. 5: ${ }^{1}$ HNMR spectrum for compound (VIc) 


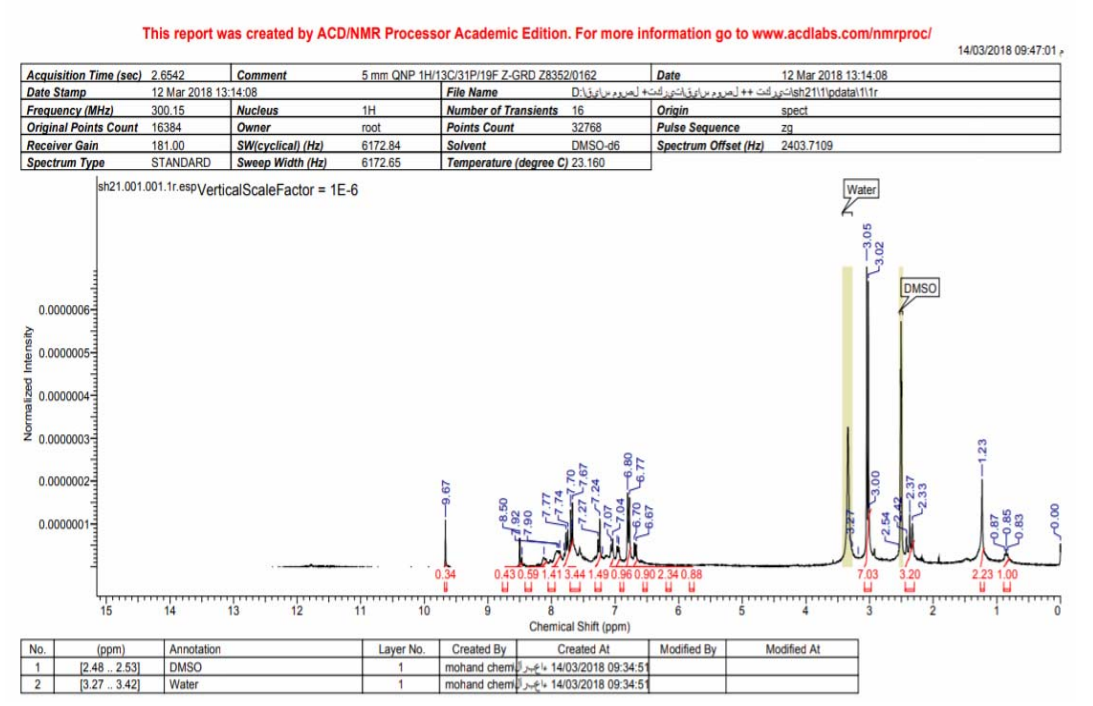

Fig. 6: ${ }^{1}$ HNMR spectrum for compound (VIId)

\section{REFERENCES}

Acheson, R.M.; Bottom, R.G. (1975). Synthesis of some acridines and 9-Acridones for spectral studies. J. C. S. Perkin., (I),650-653.

Al-Aqeel, S.I. (2017). Synthetic approaches to benzimidazoles from o-phenylene diamine: A literature review. J. Saudi Chem. Soc., 21(2), 229-237.

Al-Luhaibe, Sh. Kh. (2012). Synthesis of some New Acridine Derivatives. MSc. Thesis in Organic chemistry.

Al-Suwaidi, A.; Ahmed, H. (2010). Determination of para-phenylenediamine (PPD) in Henna in the United Arab Emirates. Int. J. Environ. Res. Public Health, (7)1681- 693.

Benguzzi, S. (2007). Synthesis and characterization of divalent transition metals complexes of Schiff bases derived from o-phenylenediamine and benzoylacetone and related species. J. Sci. and its Applications, 1(1), 79-90.

Boghaei, D.; Mohebi, S. (2002). Non-Symmetrical tetradentate vanadyl Schiff base complexes derived from 1,2-phenylenediamine and 1,3-naphthalenediamine as catalysts for the oxidation of cyclohexene. Tetrahedron. 58(26), 5357-5366.

Chong, H.; Reenak, N.; Kohry, N.; Chye, S. (2016). p-Phenylenediamine containing hair dye: An overview of Mutagenicity, Carcinogenicity and Toxicity. J. Environm. Analytical Toxicol., 5(6).

Ed Rietchel, R.L.; Flowler, J.F.; Lippin, C. (2001). Fisher contact dermatitis. Allergy to paraphenylenediamine.

El-Ajaily, M.; Abou-Krisha, M.; Etorki, A.; Al-assbaly, F.; Maihub, A. (2013). Schiff base derived from phenylenediamine and salicyaldehyde as precursor techniques in coordination chemistry. J. Chem. and Pharmaceutical Research. 5(12), 933-938

Fasina, T.; Ogundele, O.; Ayeni, I. (2014). Synthesis and biological properties of $\mathrm{N}_{2} \mathrm{O}_{2}$ Schiff bases derived from o-phenylenediamine and substituted salicylaldehydes. J. Chemical and Pharmaceutical Research. 6(6), 816- 819 (Niegeria).

Fasina, T.; Ogundele, O.; Ejiaj, F.; Dueke-Eze, C. (2012). Biological activity of copper(II), cobalt(II), and nickel (II) complexes of Schiff base derived from o- Phenylenediamine and 5-bromsalicyaldehyde. International J. Biolog. Chem., 1(6), 24-30.

Gabala, A.; Asker, M.; Barakat, A. (2007). Synthesis, characterization and biological of some platinum (II) complexes with Schiff bases derived from Salicylaldehyde, 2-furaldehyde and phenylenediamine. Spectro. Chim. Acta A mol activity Biomol Spectro. Sci., 1(67), 114-121. 
Gayathri, N.; Suresh, M. (2017). Synthesis and spectral characterization of oxova-nadium complexes derived from benzoine, o-phenylenediamine and ethylendiamine and their antibacterial activities. International J. Curr. Reas. Chem. Pharma. Sci., 1, 12-19.

Genjall, M.; Deltari, A.; Norcouzi, P.; Niasari, M.; Rabbani, M. (2004). Novel lanthanum (III) membrane sensor based on a new N-S Schiff's base. Sensors and Actuators B: Chem., 1 (28), 92-96.

Gupta, K.; Chand, A. (2003). Synthesis of polymer anchored N,N'-bis(3-allylsalidene)-ophenylenediamine cobalt(II) Schiff base complex and its catalytic activity for decomposition of hydrogen peroxide. J. Molecular Catalysis A: Chem., (202), (1-2), 253268.

Hamil, A.; Khalifa, K.; Houni, A.; Elajaily, M. (2009). Synthesis, spectroscopic investigation and antiactivity of Schiff base complexes of cobalt(II) and copper (II) ions. Rasayan J. Chem. 2(2), 261-266.

Kowaleska, M.G.; Cholewin'ski, G.; Dzierzbika, K. (2017). Recent developments in the synthesis and biological activity of acridine/ acridone analogues. Royal Soc. Chem., 7,15776

Lang, X.; Li, L.; Chen, Y.; Sun, Q.; Wu, Q.; Liu, F.; Tan, Ch.; Liu, H.; Gao, Ch.; Jiang, Y. (2013). Novel synthetic acridine derivatives as potent DNA-binding and apoptosis including antitumor agents. Bioorganic Medic. Chem., (21), 470-477.

Mahmood, A.M. (2013). Synthesis of some Heterocyclic Compounds from N-Aryl and N- Pyridyl Anthranilic Acid Derivatives. Ph.D. Thesis in Organic Chemistry.

Panda, S.; Nayak, S.S. (2009). Inclusion complexes of acridone and its semecarbazone derivatives with B-cyclodextrine: a thermodynamic spectral and antimicrobial study. Asian $J$. Research in Chem., 4(2), 539-543.

Parikh, V.M. (1974). "Absorption Spectroscopy of Organic Molecules". Addison Wesley Publishing Company, pp. 13, 46,142.

Pati, S. (1970). "The Chemistry of Carbone-nitrogen Double Bond". John Wiley and Sons, NY, 68 p.

Pethy, B.; Hohmann, J.; Minorics, R.; Varge, A.; Ocsoveski, I.; Molnar, J.; Juhasz, K.; Falkay, G.; Zupko, I. (2008). Antitumour properties of acrid one alkaloids on a murine lymphoma cell line. Anticancer Research (28), 2737- 2744) (Hungary).

Rodrigue, M.I. (2006). Novel tacrine melatonin hybirds are Dual-acting drugs for Alzheimer disease with improved acetylcholines sterase inhibitory and antioxidant properties. J. Med. Chem. (49), 59-462.

Shekouhy, A.H.; Zare, A.; Izadeh, M.R. (2008). Oxalic acid as an efficient, cheap and reusabls catalyst for the preparation of quinoxalines via condensation of 1,2-diamines with $\alpha$-diketones a troom temperature. Arkivok. XIII, 28-35.

Sondhi, S.M.; Nidhi, S.; Verma, R.P. (1997). Synthesis, anti inflammatory and analgesic activity evaluation of some 2-(9- acridinyl amino) pyridines and 2-(9-acridinyl amino/imino) thiazolines. J. Ind. Chem., 36(13), 62.

Sondhi, S.M.; BahatacharJee, G.; Jameel, R.K.; Rakesh, S.; Ran, R.; Oliver, L.; Laurent, M., Tomar, V.; Kamaluddin, G.B.; Kumar, M. (2004). Canti-inflamentry, analgesic and kinase inhibition activities of some acridine derivatives cent. Eur. J. Chem., 2(1).

Srivastava, K.; Puri, S.K (2010). Synthesis of new chalcon derivatives, containing acridinyl moiety with potential antimalarial activity. European J. Medic. Chem., (45), 745-751. 